\title{
Upstream therapeutic strategies of Valsartan and Fluvastatin on Hypertensive patients with non-permanent Atrial Fibrillation (VF-HT-AF): study protocol for a randomized controlled trial
}

\author{
Wen-Wei Qi, Tong Liu, Gang Xu, Li-Feng Li, Ying-Zi Liang, Lan Ye and Guang-Ping Li
}

\begin{abstract}
Background: Previous studies regarding rhythm control in patients with atrial fibrillation (AF) could not sufficiently demonstrate the efficacy of available anti-arrhythmic drugs. 'Upstream therapy' has emerged as a potential strategy for the prevention and treatment of AF. The use of angiotensin II receptor blockers and statins has been suggested to decrease new-onset AF, but which remains inadequately explored. This study was designed to examine whether valsartan or fluvastatin can reduce the risk of non-permanent AF in patients with hypertension.

Methods/design: The VF-HT-AF study is a multicenter, randomized, open-label, four-arm parallel group study with comparative evaluation of valsartan and fluvastatin as upstream therapies for the treatment of non-permanent AF complicated by hypertension. The primary outcome measure is change in the development of paroxysmal AF into persistent or permanent AF, the development of persistent AF to permanent AF, and change in incidence of overall and persistent AF recurrence, as evaluated by 7-days ambulatory electrocardiograph monitoring (Holter) and patients' diaries during 2 years' follow-up. Secondary outcome measures of this study include the occurrence of: (1) fatal and nonfatal myocardial infarction; (2) heart failure (New York Heart Association stage III or IV); (3) cardiogenic shock; (4) serious bleeding necessitating hospitalization; (5) malignant ventricular arrhythmia; (6) revascularization therapy; (7) radiofrequency catheter ablation of AF; (8) changes of left atrial dimension, as measured by ultrasound echocardiography; (9) stroke; (10) cardiovascular mortality; and (11) all-cause mortality. A total of 1879 patients will be investigated from 15 medical centers throughout China to obtain the relevant information.
\end{abstract}

Discussion: This is the first study in hypertensive patients complicated non-permanent AF in the Chinese population. Results of this study will inform the use of upstream therapies of AF.

Trial registration: chictr.org, ChiCTR-TRC-12002642

Keywords: 7-day Holter, Atrial fibrillation, Fluvastatin, Randomized controlled clinical trial, Valsartan

\section{Background}

Atrial fibrillation (AF), the most common clinically significant cardiac arrhythmia, is associated with increased mortality and morbidity $[1,2]$, especially in hypertensive patients [3]. In general, hypertension is the most important risk factor for AF. Although much effort has been put into the development of an effective

\footnotetext{
* Correspondence: tjcardiol@tijmu.edu.cn

Tianjin Key Laboratory of Ionic-Molecular Function of Cardiovascular disease, Department of Cardiology, Tianjin Institute of Cardiology, Second Hospital of Tianjin Medical University, Tianjin 300211, PR China
}

pharmacological treatment, several trials conducted in the USA and Europe proved that existing traditional anti-arrhythmic drugs failed to improve the prognosis of patients with AF [4-7].

Several systematic reviews and meta-analyses have confirmed anti-arrhythmic efficacy while raising concerns about adverse events and mortality [8]. In the past few years, a number of trials investigating upstream therapy for the prevention of AF have been reported [9]. Upstream therapies are long-term modulators of atrial remodeling regarding structure or function, which may change 
molecular expression contributing to the arrhythmia. The renin-angiotensin system (RAS) is an important therapeutic target for atrial remodeling. Angiotensin II receptor blockers (ARBs) have recently been reported to suppress AF recurrence in both paroxysmal and persistent AF in selected patients. However, recent placebo-controlled, double-blind trials with ARBs failed to show convincing results $[10,11]$. Statins, as a well-established secondary prevention benefit for atherosclerotic coronary artery disease, are hypothesized to be beneficial against atrial arrhythmia, yet the data are inconsistent [12-15]. In addition, there have been little prospective and randomized studies to evaluate the effectiveness of upstream therapeutic strategies in hypertensive patients with nonpermanent AF.

To confirm the effectiveness of ARBs or statins on AF in hypertensive patients, the Upstream Therapeutic Strategies of Valsartan and Fluvastatin on Hypertensive Patients with Non-permanent AF (VF-HT-AF study) will be conducted, with 1879 participants from 15 centers.

\section{Methods/design}

The VF-HT-AF study is designed as a prospective, randomized, open-label, four-arm parallel, and multicenter study. The objective of the study is to test the hypothesis that upstream therapy using valsartan or fluvastatin is more effective in reducing the recurrence of $\mathrm{AF}$ and the progress from non-permanent $\mathrm{AF}$ to permanent $\mathrm{AF}$ in hypertensive patients with non-permanent AF, compared with conventional antihypertensive therapy using dihydropyridine calcium channel blockers (CCBs). This study will be conducted according to the principles outlined in the Declaration of Helsinki. Written informed consent will be obtained from all patients prior to the study. The study protocol has been approved by the ethics committee of the Second Hospital of Tianjin Medical University (Clinical ethical review, 2012, No. 27) as well as other participating medical centers, which are secondary or tertiary healthcare providers in China, and should provide proof of laboratory quality control. The ethical bodies that approved our study in the various centers are listed in Additional file 1. If there is any amendment to the protocol, approval must again be sought from the ethics committee. The study is registered with the Chinese Clinical Trial Registry (ChiCTR-TRC-12002642). The protocol design is based on the Consolidated Standards of Reporting Trials (CONSORT) and Standard Protocol Items: Recommendations for Interventional Trials (SPIRIT, see Additional file 2), and study results will be reported according to these guidelines.

\section{Patient population and entry criteria}

Patients enrolled in the study need to meet the inclusive recruited criteria: (1) have hypertension, defined as an average systolic blood pressure $\geq 140 \mathrm{mmHg}(1 \mathrm{~mm} \mathrm{Hg}=0.133$ $\mathrm{kPa}$ ) or a diastolic blood pressure $\geq 90 \mathrm{mmHg}$ (but a systolic blood pressure $<180 \mathrm{mmHg}$ and a diastolic blood pressure $<110 \mathrm{mmHg}$ ) at the first visit, or the requirement of any anti-hypertension treatment at enrollment; (2) a history of non-permanent AF within 1 year prior to the enrollment, as confirmed by electrocardiography (ECG), and converted to sinus rhythm; (3) have not taken ARBs or angiotensin-converting enzyme inhibitors (ACEIs) as well as statins in the previous 2 weeks, or are taking ARBs, ACEIs and statins, but can accept a 2-week washout period; (4) aged 25-79 years; and (5) willing to sign the informed consent form (Additional file 3).

The exclusion criteria are: (1) persistent AF with a duration $\geq 1$ year or permanent AF; (2) serious left main coronary artery disease identified by coronary angiography; (3) heart failure (New York Heart Association [NYHA] stage III or IV); (4) acute myocardial infarction in the previous 3 months; (5) surgical or interventional indications of valvular heart disease; (6) uncontrolled thyroid disease (abnormal free T3, free T4, or thyroid stimulating hormone, or requiring any anti-thyroid treatment at enrollment); (7) serious liver or renal dysfunction (ALT $>80 \mathrm{U} / \mathrm{l}$, AST $>80 \mathrm{U} / \mathrm{l}$, or creatinine $>132$ $\mu \mathrm{mol} / \mathrm{l})$; (8) history of unstable angina pectoris; (9) stroke or transient ischemic attack within the previous 3 months; (10) poor treatment compliance, such as central nervous system or mental illness, or a possibility of being uncooperative in the follow-up period; (11) have taken ARBs, ACEIs or statins but cannot accept a 2week washout period; (12) obvious hyperlipidemia that must be treated by statins or fibrates; (13) contraindication of statins or ARBs; (14) pregnancy or the possibility of pregnancy, or breast feeding; and (15) aged younger than 25 or older than 79 years.

\section{Study design}

The VF-HT-AF study is a randomized, multicenter, openlabel, and four-arm parallel study to evaluate the effectiveness of valsartan, fluvastatin or a combination of both on recurrent $\mathrm{AF}$ in hypertensive patients with nonpermanent AF in China. After providing informed consent, patients will be randomly assigned using a computer system to one of four groups (the valsartan group, the fluvastatin plus dihydropyridine CCBs group, the valsartan plus fluvastatin group, and the dihydropyridine CCBs group) by the School of Public Health of Tianjin Medical University, initially in a 1:1:1:1 ratio. Physicians, who should be qualified medical practitioners, screen and follow up the patients in hospital outpatient or inpatient clinics. All patients' data will be collected using a standard case report form and transmitted to the central database at the data center. All centers will be regularly monitored for 
source data documentation. Missing or questionable data will be completed and corrected by queries.

If the candidate patients have not taken ARBs, ACEIs or statins in the past at least 14 days, they will be directly enrolled and randomized. If the candidate patients have taken ARBs, ACEIs or statins, they will be randomized after a 14-day washout period. Baseline data will be collected after the washout period.

The initial dose of fluvastatin will be $40 \mathrm{mg} /$ day at night, while valsartan will be prescribed initially at 80 $\mathrm{mg} /$ day. The fluvastatin dose will be no less than $40 \mathrm{mg} /$ day and that of valsartan will be no less than $80 \mathrm{mg} /$ day during the follow-up period. Blood pressures in each group must reach the target blood pressure, which is set at $<140 / 90 \mathrm{~mm} \mathrm{Hg}$.

\section{Follow-up}

Clinical follow-up using standardized questionnaires will be performed every 3 months during the 2 years followup period from the assignment of each patient.

The dosages and types of all other anti-hypertensive, anti-hyperlipidemia, and anti-arrhythmic drugs used during the follow-up will be recorded for each patient. Ultrasound echocardiography will be performed before the patients enter the study and at the end of the followup period. 7-day Holter monitoring will be performed at the baseline, 6 month, 12 month and at the end of the follow-up period. Patients' diaries, cardiac function
(NYHA classification) and adverse events during the follow-up period will be collected every 3 months.

Throughout the study, patients should record diaries when they feel discomfort. Arrhythmia-related symptoms are self-evaluated or evaluated by 7-day Holter monitoring, which has five poles, is weighted $50 \mathrm{~g}, 2.0 \times 2.7 \mathrm{~cm}$, and can record ECG for 7 days. The 7-day Holter instruments were manufactured by BORSAM Co. in Shenzhen, Guangdong Province, China, and provided by the study's sponsor, Tianjin Institute of Cardiology, Tianjin, China. The attending physicians will also record blood pressure and ECG during each follow-up. Unless necessary, anti-arrhythmic drug therapy will be discontinued during the study according to the attending physicians' advice for each patient. The design of the study is shown in Fig. 1.

\section{Compliance strategy}

To maximize subjects' compliance, first, we will have a thorough consent process for all participants. We will explain the schedule, potential side effects of treatment, and the responsibilities of the subjects in detail. Second, we will try to prevent dropouts by providing ongoing support to patients. A direct telephone line set up for this trial will enable the study team to communicate with the patients personally. An information sheet will be given to each participant providing them or their caregivers with means of urgent contact. Extra visits and

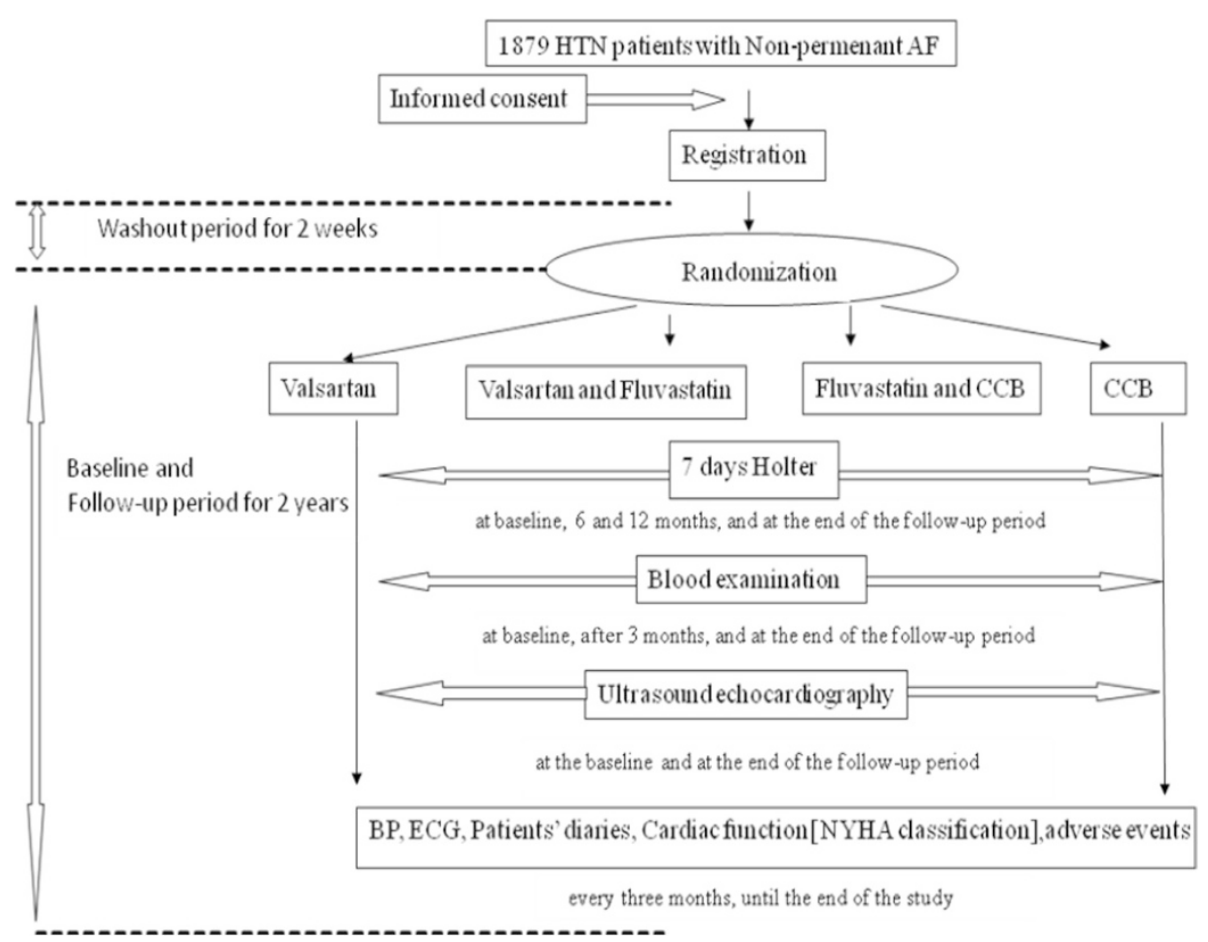

Fig. 1 Flow-process diagram. BP, blood pressure; ECG, electrocardiography 
free medical care will be arranged for any participant who feels harmed by the trial protocol. Certainly those participants in the control group should not experience any difference in their condition.

\section{Primary outcome measures}

The VF-HT-AF study will examine whether fluvastatin or valsartan has anti-arrhythmic effects on non-permanent $\mathrm{AF}$, apart from their lipid-lowering and antihypertensive effects, in comparison with dihydropyridine CCBs. Also, it will confirm whether a combination of valsartan and fluvastatin has better effects on non-permanent AF than either drug alone.

Therefore, the primary outcome measure is the change in the development of paroxysmal AF into persistent or permanent $\mathrm{AF}$, the development of persistent $\mathrm{AF}$ to permanent AF, as well as the change in incidence of overall and persistent AF recurrence, which will be evaluated by 7-day Holter monitoring and patients' diaries during the 2-year's follow-up of the four treatment groups.

\section{Secondary outcome measures}

The secondary outcome measures of this study include the occurrence of: (1) myocardial infarction; (2) heart failure (NYHA III or IV); (3) cardiogenic shock; (4) serious bleeding necessitating hospitalization; (5) malignant ventricular arrhythmia (including ventricular tachycardia or fibrillation); (6) revascularization therapy (coronary artery bypass graft or percutaneous coronary interventions); (7) radiofrequency catheter ablation of AF; (8) changes of left atrial dimension, as measured by ultrasound echocardiography; (9) stroke; (10) cardiovascular mortality; and (11) all-cause mortality.

\section{Definitions}

The definitions of AF are made according to 2012 HRS/EHRA/ECAS Expert Consensus [16]. Paroxysmal $\mathrm{AF}$ is defined as recurrent AF (at least two episodes) that terminates spontaneously within 7 days. Episodes of AF no more than 48 hours' duration that are terminated with electrical or pharmacologic cardioversion should also be classified as paroxysmal AF episodes. Persistent AF is defined as continuous AF that is sustained beyond 7 days. Episodes of AF lasting more than 48 hours, but less than 7 days, in which a decision is made to cardiovert AF electrically or pharmacologically should also be classified as persistent AF episodes. Permanent AF refers to long-lasting episodes of AF that could be considered by any evidence as lasting longer than 1 year, or to a case of AF for which a decision has been made not to restore or maintain sinus rhythm by any means, including catheter ablation or surgery. Myocardial infarction is defined as a cardiac troponin rise accompanied by typical symptoms, pathological $\mathrm{Q}$ waves, ST elevation or depression, or coronary intervention. Heart failure (NYHA III or IV) requires echocardiographic evidence of reduced left ventricular ejection fraction or a diagnosis of heart failure by a cardiologist. Cardiogenic shock is defined by sustained hypotension with tissue hypoperfusion despite adequate left ventricular filling pressure. Signs of tissue hypoperfusion include oliguria ( $<30 \mathrm{ml} /$ hour$)$, cool extremities, and altered level of consciousness.

\section{Sample size calculation}

The primary outcome measure is the change in the development of paroxysmal AF into persistent or permanent $\mathrm{AF}$, the development of persistent $\mathrm{AF}$ to permanent $\mathrm{AF}$, as well as the change in incidence of overall and persistent AF recurrence. Power calculations to provide estimates for the necessary sample size were conducted concerning the primary outcome measure. The sample size was estimated based on findings from the 12-month results of the J-RHYTHM II study [11], in which the incidence of persistent AF is estimated to be $6.8 \%$ lower in the valsartan group than in the CCBs group. To ensure $80 \%$ power at the $5 \%$ significance level, a sample size of at least 1404 needs to be included. Considering a rejection rate of $20 \%$, about 1879 patients need to be approached.

\section{Data management}

All the data will be entered and stored in a passwordprotected computer. To ensure high quality of the data, a double data entry method will be used. A data monitoring committee, of which at least two members will be independent of the research team, will monitor the data management process regularly. All the data will be frozen and then locked to prevent further editing after the validation by the data monitoring committee. Only the data monitoring committee, the study research assistant and the principal investigator will have access to the final data set. The protocol and statistical results will be published in a scientific journal.

\section{Statistical analysis}

All analyses will be performed based on the intentionto-treat principle with differences assumed to be significant at a two-sided $P$ value $<0.05$.

Baseline characteristics and follow-up information will be collected for each group through the questionnaire. Absolute values for each question will be used to calculate the mean value, standard deviation, median value, percentile, number of cases, and percentages per group. Statistical significance will be evaluated by analysis of variance or Kruskal-Wallis rank sum test for continuous variables and chi-squared test for categorical variables, respectively. Kaplan-Meier curves will be used to describe 
the time-dependent occurrence of events, and the logrank test will be performed to compare survival distributions for the four groups. To adjust for possible baseline imbalances between groups, a Cox proportional hazards model will be used. A hazard ratio will be calculated. In addition, subgroup analyses will be performed corresponding to the nature of the data. All statistical analyses will be performed using SPSS statistical software (version 17.00, Chicago, IL, USA).

\section{Quality assurance}

\section{Steering committee}

The intervention of the steering committee includes general practice training and practice visits, to develop and monitor the implementation of the protocol. Telephone support is delivered by the intervention team with assistance from the principle investigators. The quality of the intervention process will be monitored and assured by a steering committee using multiple strategies, including a standardized selection, training and performance assessments of the intervention team, evaluation of general practice training, records of practice visits kept by the intervention team, and ongoing feedback by practice staff on the intervention during the 24-month period. The steering committee will be supported by a statistician, who is responsible for ensuring the timely publication of this study results.

\section{7-day Holter diagnosis committee}

This committee will determine the rhythm of the 7-day Holter ECGs without knowing the patients' conditions and will be responsible for the diagnosis of the recorded 7-day Holter ECGs before the analysis of the primary and secondary outcome measures.

\section{Safety monitoring board}

The safety monitoring board will be responsible for monitoring patient safety and will recommend premature cessation of the trial should there be an increase in unpredicted adverse events.

\section{Discussion}

Paroxysmal AF often progresses to persistent or permanent $\mathrm{AF}$ when the former increases in frequency and duration, and accounts for approximately $5.5 \%$ of all patients with paroxysmal AF per year [17]. One consistent fact that emerges from studies is that conventional anti-arrhythmic agents, such as class I antiarrhythmics, do not improve survival rates [18], and their efficacy in preventing progression of paroxysmal AF to persistent AF is limited because they do not exert any potential benefits on electrical or structural remodeling, which contributes to the pathophysiological basis of AF [19].
Preventing new-onset and recurrent AF with upstream therapies is of great interest, but current data are conflicting. Further studies are needed to optimize rhythm control by anti-arrhythmic drugs and targeted catheter ablation to specific patient populations at an earlier stage. There is little data on valsartan and fluvastatin treatment in patients with hypertension and nonpermanent AF.

\section{Stains: fluvastatin}

During recent years, statins have emerged as one of the most effective treatments to reduce the burden of cardiovascular disease worldwide. Owing to their remarkably good safety profile and declining costs, there has been some interest in the potential use of statins as direct anti-arrhythmic or anti-inflammatory drugs [20]. Meta-analysis demonstrated that the use of statins was significantly associated with a decreased risk of AF in patients with sinus rhythm [12, 15, 21, 22]. Statins could be considered for patients with intermediate risk factors [23] as a secondary prevention of AF [22]. These results provided some evidence for the benefit of statins beyond their lipid-lowering activity [20].

The potential mechanisms involved in AF reduction associated with statin therapy are not fully clear. One possible pathway involves inflammation, which has been recognized as an accomplice and a potential trigger for AF [24]. Bellosta et al. [25] have suggested that statins have pleiotropic properties, and their anti-inflammatory effects are associated with a reduction in the expression of cytokines, intercellular adhesion molecules, and interleukins. Statins reduced the incidence of paroxysmal AF with a concomitant decrease in C-reactive protein levels [26], which are believed to be a risk factor for AF. Statin treatment reduced inflammatory biomarkers, which might also explain a potentially beneficial effect of statins against AF [27]. More recently, antioxidative actions have been hypothesized to prevent electrical remodeling [28]. The administration of statins significantly decreased generation of reactive oxygen species in vitro and in vivo [29]. Statins prevented the development of AF by modulating extracellular remodeling. Statins modified extracellular components by regulating the expression of matrix metalloproteinases or their inhibitors [30]. Moreover, statins might downregulate the RAS and modulate autonomic nervous systeminduced increases in sympathetic activity, which has been shown to promote atrial remodeling. Other potential mechanisms of action include modification of atherosclerotic plaque [31], improvement of endothelial function [32], and alteration of membrane fluidity and ion channel conductance [21].

Some clinical and experimental studies have suggested the use of statins to protect against AF [33]. However, insufficient data are available to allow the recommendation 
of statins for the prevention of AF [34]. The STOP AF trial [27] showed that high-dose atorvastatin did not reduce the recurrence of AF after cardioversion. Lee et al. [35] have suggested that statin therapy in patients with paroxysmal AF might be limited to the prevention of incident $\mathrm{AF}$, but it does not appear to inhibit the progression of paroxysmal AF to permanent AF.

Although some studies [36] established diverse results, they concur that statins are beneficial in decreasing the frequency of paroxysmal AF. We are, however, unable to conclude whether a benefit exists with the use of statins in inhibiting the progression of paroxysmal to persistent $\mathrm{AF}$, owing in part to the limited data available.

Interestingly, fluvastatin was able to confer benefit, despite having the lowest potency. An advantage of fluvastatin is its long-acting formation, which the DECREASE study investigators stated could serves as a 'bridge' during the post-operative period, when patients were not receiving oral medications. For practice to reflect clinical trials, atorvastatin would be selected before percutaneous coronary intervention and coronary artery bypass graft, whereas fluvastatin would be selected before noncardiac surgical procedures [13]. Therefore, we selected fluvastatin as the treatment statin in our study. Because it is not clear whether the effects of statin use on AF depend on the underlying lipid levels, we will enroll patients without severe hyperlipidemia.

\section{ARBs: valsartan}

The VALUE trial [3] found that the incidence of persistent AF was $1.35 \%$ with valsartan and $1.97 \%$ with amlodipine (unadjusted hazard ratio 0.683, $95 \%$ confidence interval: $0.525-0.889, P=0.0046)$. The VALUE trial results demonstrated that valsartan-based treatment reduced the development of new-onset AF, particularly sustained AF in hypertensive patients, compared with amlodipine-based therapy. These findings suggest that ARBs might result in greater benefits than calcium antagonists in hypertensive patients at risk of AF. This leads to the question of whether or not the RAS is an underlying mechanism that can be targeted to prevent AF. Several mechanisms have been proposed to explain the effects of RAS blockade on prevention of AF, including decreased atrial stretch, lowered end-diastolic left ventricular pressure and left atrial pressure, prevention of atrial fibrosis, modification of sympathetic tone, alteration in potassium currents and atrial refractoriness, and direct antiarrhythmic effects [37-40]. However, a recent J-RHYTHM II study [11] has found that there were no significant differences between an ARB (candesartan) and a CCB (amlodipine) in the development of persistent $\mathrm{AF}$ in the treatment of paroxysmal $\mathrm{AF}$ associated with hypertension.

Therefore, large-scale, prospective, randomized clinical trials are urgently needed to establish whether statins or
ARB bring a similar benefit and are an appropriate therapeutic option in the hypertensive patients for the management of AF.

\section{7-day Holter}

The Holter monitor has been recently developed, which can perform ECG continuously without the need for removal during exercise or sleeping. However, the 24-hour Holter cannot record arrhythmia for longer than one day. To monitor AF consistently, we used the 7-day Holter system and patients' diaries to record AF in a Chinese population.

The VF-HT-AF study is a prospective, randomized, open-labeled, multicenter trial designed to provide novel data necessary to comprehensively assess the efficiency of valsartan and fluvastatin on AF prevention in patients with hypertension and non-permanent $\mathrm{AF}$.

\section{Expected implications}

The VF-HT-AF study will determine the anti-arrhythmic effects of the ARB and statin on the recurrence of nonpermanent $\mathrm{AF}$ associated with hypertension. It will also examine the mortality, morbidity, and perpetuation of the arrhythmia under valsartan, fluvastatin, dihydropiridine CCBs or combined treatment.

In particular, prevention of persistent and permanent $\mathrm{AF}$ would be expected to reduce hospitalization for heart failure, the incidence of stroke, morbidity, and mortality in patients with hypertension. This study will provide data to health care policymakers and the authors of clinical guidelines regarding the appropriate use of upstream therapy in hypertensive patients with $\mathrm{AF}$ and provide directions for future research.

Therefore, the results of this study will emphasize the role of upstream therapies in the prevention of AF in a Chinese hypertensive population and assist in the design of an optimal therapy for such patients.

However, one important limitation that might be present in this study is that it is an open-label trial, because we do not have enough funds to make the drugs in blinded versions. The drugs that the patients will use in our study are all provided in hospitals or pharmacies. The patients and doctors will know the drugs used. Another limitation is that the control group is not blank, so we do not test the fluvastatin effect alone. Because the patients in our study are hypertensive patients, they must, for ethical reasons, be treated with antihypertensive therapy. We chose the group receiving dihydropyridine $\mathrm{CCBs}$ as the control group, comparing them with the dihydropyridine CCBs plus fluvastatin group. If the recurrence of AF in the dihydropyridine CCBs plus fluvastatin group is less than the dihydropyridine CCBs group, it will be proved that fluvastatin can reduce the probability of non-permanent $\mathrm{AF}$ in 
patients with hypertension. There may be some combined effects of dihydropyridine CCBs plus fluvastatin, but we have to use antihypertensive drugs.

\section{Trial status}

The trial is currently in the recruitment phase.

\section{Additional files}

\section{Additional file 1: Ethical bodies that approved the study. Additional file 2: SPIRIT-Checklist 2013. \\ Additional file 3: Informed consent statement.}

\section{Abbreviations}

ACEl: angiotensin-converting enzyme inhibitor; AF: atrial fibrillation; ARB: angiotensin II receptor blocker; CCB: calcium channel blocker; CONSORT: Consolidated Standards of Reporting Trials; ECG: Electrocardiography; NYHA: New York Heart Association; RAS: renin-angiotensin system; SPIRIT: Standard Protocol Items: Recommendations for Interventional Trials.

\section{Competing interests}

The authors declare that they have no competing interests.

\section{Authors' contributions}

WWQ participated in the study design, drafted the manuscript, obtained ethical approval, planned the statistical analysis, and revised the manuscript. TL participated in the study conception and design, and contributed to revising the manuscript. GX and LFL participated in the study conception and design. YZL is the head of the Steering Committee. LY participated in the study design and is the head of the 7-day Holter diagnosis committee. GPL, as the principal investigator, conceived the study, drafted the manuscript, obtained ethical approval, and reviewed the manuscript. All authors read and approved the final manuscript.

\section{Acknowledgements}

The VF-HT-AF study is funded by the Tianjin Municipal Science and Technology Commission, China. This study is sponsored by the Tianjin Institution of Cardiology, Second Hospital of Tianjin Medical University, and support in the data analysis is provided by the School of Public Health of Tianjin Medical University. All study designs and protocols of the VF-HT-AF study have been approved by the ethics committee of the Second Hospital of Tianjin Medical University.

Received: 31 July 2014 Accepted: 1 July 2015

Published online: 07 August 2015

\section{References}

1. Fuster V, Ryden LE, Cannom DS, Crijns HJ, Curtis AB, Ellenbogen KA, et al. 2011 ACCF/AHA/HRS focused updates incorporated into the ACC/AHA/ESC 2006 guidelines for the management of patients with atrial fibrillation: a report of the American College of Cardiology Foundation/American Heart Association Task Force on practice guidelines. Circulation. 2011;123:269-367.

2. Benjamin EJ, Chen PS, Bild DE, Mascette AM, Albert CM, Alonso A, et al. Prevention of atrial fibrillation: report from a national heart, lung, and blood institute workshop. Circulation. 2009:119:606-18.

3. Schmieder RE, Kjeldsen SE, Julius S, McInnes GT, Zanchetti A, Hua TA. Reduced incidence of new-onset atrial fibrillation with angiotensin II receptor blockade: the VALUE trial. J Hypertens. 2008;26:403-11.

4. Wyse DG, Waldo AL, DiMarco JP, Domanski MJ, Rosenberg Y, Schron EB, et al. A comparison of rate control and rhythm control in patients with atrial fibrillation. N Engl J Med. 2002:347:1825-33.

5. Van Gelder IC, Hagens VE, Bosker HA, Kingma JH, Kamp O, Kingma T, et al. A comparison of rate control and rhythm control in patients with recurrent persistent atrial fibrillation. N Engl J Med. 2002;347:1834-40.
6. Hohnloser $\mathrm{SH}, \mathrm{Kuck} \mathrm{KH}$, Lilienthal J. Rhythm or rate control in atrial fibrillation - Pharmacological Intervention in Atrial Fibrillation (PIAF): a randomised trial. Lancet. 2000;356:1789-94.

7. Carlsson J, Miketic S, Windeler J, Cuneo A, Haun S, Micus S, et al. Randomized trial of rate-control versus rhythm-control in persistent atrial fibrillation: the Strategies of Treatment of Atrial Fibrillation (STAF) study. J Am Coll Cardiol. 2003;41:1690-6.

8. Lafuente-Lafuente C, Mouly S, Longas-Tejero MA, Mahe I, Bergmann JF. Antiarrhythmic drugs for maintaining sinus rhythm after cardioversion of atrial fibrillation: a systematic review of randomized controlled trials. Arch Intern Med. 2006;166:719-78.

9. Savelieva I, Kakouros N, Kourliouros A, Camm AJ. Upstream therapies for management of atrial fibrillation: review of clinical evidence and implications for European Society of Cardiology guidelines. Part I: primary prevention. Europace. 2011;13:308-28.

10. Goette A, Schon N, Kirchhof P, Breithardt G, Fetsch T, Hausler KG, et al. Angiotensin II-antagonist in paroxysmal atrial fibrillation (ANTIPAF) trial. Circ Arrhythm Electrophysiol. 2012;5(1):43-51

11. Yamashita T, Inoue H, Okumura K, Kodama I, Aizawa Y, Atarashi H, et al. Randomized trial of angiotensin II-receptor blocker vs. dihydropiridine calcium channel blocker in the treatment of paroxysmal atrial fibrillation with hypertension (J-RHYTHM II study). Europace. 2011;13:473-9.

12. Liu T, Li L, Korantzopoulos P, Liu E, Li G. Statin use and development of atrial fibrillation: a systematic review and meta-analysis of randomized clinical trials and observational studies. Int J Cardiol. 2008;126:160-70.

13. Winchester DE, Wen X, Xie L, Bavry AA. Evidence of pre-procedural statin therapy a meta-analysis of randomized trials. J Am Coll Cardiol. 2010:56:1099-109.

14. Rahimi K, Emberson J, McGale P, Majoni W, Merhi A, Asselbergs FW. Effect of statins on atrial fibrillation: collaborative meta-analysis of published and unpublished evidence from randomised controlled trials. BMJ. 2011;342:1250

15. Liu T, Korantzopoulos P, Li L, Li G. Preventive effects of rosuvastatin on atrial fibrillation: a meta-analysis of randomized controlled trials. Int J Cardiol. 2013;167:3058-60.

16. Calkins H, Kuck KH, Cappato R, Brugada J, Camm AJ, Chen SA, et al. 2012 HRS/EHRA/ECAS expert consensus statement on catheter and surgical ablation of atrial fibrillation: recommendations for patient selection, procedural techniques, patient management and follow-up, definitions, endpoints, and research trial design: a report of the Heart Rhythm Society (HRS) Task Force on Catheter and Surgical Ablation of Atrial Fibrillation Heart Rhythm. 2012;9:632-96

17. Kato T, Yamashita T, Sagara K, linuma H, Fu LT. Progressive nature of paroxysmal atrial fibrillation: observations from a 14-year follow-up study Circ J. 2004;68:568-72.

18. Corley SD, Epstein AE, DiMarco JP, Domanski MJ, Geller N, Greene HL, et al. Relationships between sinus rhythm, treatment, and survival in the Atrial Fibrillation Follow-Up Investigation of Rhythm Management (AFFIRM) Study. Circulation. 2004:109:1509-13.

19. Wijfels MC, Kirchhof CJ, Dorland R, Allessie MA. Atrial fibrillation begets atrial fibrillation: a study in awake chronically instrumented goats. Circulation. 1995;92:1954-68.

20. Fang WT, Li HJ, Zhang H, Jiang S. The role of statin therapy in the prevention of atrial fibrillation: a meta-analysis of randomized controlled trials. Br J Clin Pharmacol. 2012;74:744-56.

21. Fauchier L, Pierre B, de Labriolle A, Grimard C, Zannad N, Babuty D. Antiarrhythmic effect of statin therapy and atrial fibrillation a meta-analysis of randomized controlled trials. J Am Coll Cardiol. 2008;51:828-35.

22. Fauchier L, Clementy N, Babuty D. Statin therapy and atrial fibrillation: systematic review and updated meta-analysis of published randomized controlled trials. Curr Opin Cardiol. 2013;28:7-18.

23. Fleisher LA, Beckman JA, Brown KA, Calkins H, Chaikof EL, Fleischmann KE, et al. ACC/AHA 2007 guidelines on perioperative cardiovascular evaluation and care for noncardiac surgery. J Am Coll Cardiol. 2007;50:1707-32.

24. Liu T, Li G, Li L, Korantzopoulos P. Association between C-reactive protein and recurrence of atrial fibrillation after successful electrical cardioversion: a meta-analysis. J Am Coll Cardiol. 2007:49:1642-8.

25. Bellosta S, Ferri N, Bernini F, Paoletti R, Corsini A. Non-lipid-related effects of statins. Ann Med. 2000;32:164-76

26. Dernellis J, Panaretou M. Effect of C-reactive protein reduction on paroxysmal atrial fibrillation. Am Heart J. 2005;150:1064. 
27. Negi S, Shukrullah I, Veledar E, Bloom HL, Jones DP, Dudley SC. Statin therapy for the prevention of atrial fibrillation trial (STOP AF trial). J CardiovasC Electrophysiol. 2011;22:414-9.

28. Liu T, Korantzopoulos P, Li G. Antioxidant therapies for the management of atrial fibrillation. Cardiovasc Diagn Ther. 2012;2:298-307.

29. Watanabe T, Yasunari K, Nakamura M. Antioxidative actions of statins: potential mechanisms for antiathersclerotic effects. Mini Rev Med Chem. 2006;6:505-8.

30. Marin F, Pascual DA, Roldan V, Arribas JM, Ahumada M, Tornel PL, et al. Statins and postoperative risk of atrial fibrillation following coronary artery bypass grafting. Am J Cardiol. 2006;97:55-60.

31. Okazaki S, Yokoyama T, Miyauchi K, Shimada K, Kurata T, Sato H, et al. Early statin treatment in patients with acute coronary syndrome: demonstration of the beneficial effect on atherosclerotic lesions by serial volumetric intravascular ultrasound analysis during half a year after coronary event: the ESTABLISH Study. Circulation. 2004;110:1061-8.

32. Vlachopoulos C, Aznaouridis K, Dagre A, Vasiliadou C, Masoura C, Stefanadi E, et al. Protective effect of atorvastatin on acute systemic inflammation-induced endothelial dysfunction in hypercholesterolaemic subjects. Eur Heart J. 2007;28:2102-9.

33. Kostapanos MS, Liberopoulos EN, Goudevenos JA, Mikhailidis DP, Elisaf MS Do statins have an antiarrhythmic activity? Cardiovasc Res. 2007:75:10-20.

34. Camm AJ, Kirchhof P, Lip GY, Schotten U, Savelieva I, Ernst S, et al. Guidelines for the management of atrial fibrillation: the Task Force for the Management of Atrial Fibrillation of the European Society of Cardiology (ESC). Eur Heart J. 2010;31:2369-429.

35. Lee $\mathrm{YL}$, Blaha MJ, Jones SR. Statin therapy in the prevention and treatment of atrial fibrillation. J Clin Lipidol. 2011;5:18-29.

36. Hirayama Y, Atarashi H, Kobayashi Y, Iwasaki Y, Miyauchi Y, Ohara T, et al. Long-term effects of upstream therapy on paroxysmal atrial fibrillation in patients without overt heart diseases. Int Heart J. 2009;50:141-51.

37. Ehrlich JR, Hohnloser SH, Nattel S. Role of angiotensin system and effects of its inhibition in atrial fibrillation: clinical and experimental evidence. Eur Heart J. 2006;27:512-8

38. Boos CJ, Anderson RA, Lip GY. Is atrial fibrillation an inflammatory disorder? Eur Heart J. 2006;27:136-49.

39. Engelmann MD, Svendsen $\mathrm{JH}$. Inflammation in the genesis and perpetuation of atrial fibrillation. Eur Heart J. 2005;26:2083-92.

40. Dernellis J, Panaretou M. Relationship between C-reactive protein concentrations during glucocorticoid therapy and recurrent atrial fibrillation. Eur Heart J. 2004:25:1100-7.

\section{Submit your next manuscript to BioMed Central and take full advantage of:}

- Convenient online submission

- Thorough peer review

- No space constraints or color figure charges

- Immediate publication on acceptance

- Inclusion in PubMed, CAS, Scopus and Google Scholar

- Research which is freely available for redistribution 